\title{
Vector Planning and Corneal Topographic Astigmatism
}

\author{
Noel Alpins \\ NewVision Clinics, Cheltenham, Victoria, Australia
}

DOl: https://doi.org/10.17925/EOR.2018.12.2.83

$\mathrm{T}$ e concept of planning refractive laser treatments using parameters other than manifest or wavefront refraction was somewhat of a novelty until topography-guided treatments came to the forefront more recently. However, both forms of treatment still have their limitations: manifest and wavefront refraction-based treatments do not take into account the effects on corneal astigmatism, and topography-guided treatments do not take into account any astigmatism behind the cornea or any cortical processing involved in the patient's subjective astigmatic perception. The answer to optimally correcting the astigmatism of the eye is to treat somewhere between the two extremes of refractive cylinder and corneal astigmatism. This can be done using the systematic approach of Vector Planningø (ASSORT Pty. Ltd., Cheltenham, Victoria, Australia). Vector Planning is a systematic approach designed to treat the maximum amount of astigmatism of the eye, while reducing the amount of corneal astigmatism remaining postoperatively compared to treatments based on refractive parameters alone.

\section{Keywords}

Astigmatism, Vector Planning ${ }^{\circledR}$ ocular residual astigmatism, corneal topographic astigmatism, CorT

Disclosure: Noel Alpins is the chief executive officer of ASSORT Surgical Management Systems.

Review Process: This article is a short opinion piece and, as such, did not undergo the journal's usual peer review process, but was reviewed by the editorial board before publication.

Authorship: The named author meets the International Committee of Medical Journal Editors (ICMJE) criteria for authorship of this manuscript, takes responsibility for the integrity of the work as a whole, and has given final approval for the version to be published.

open Access: This article is published under the Creative Commons Attribution Non-commercial License, which permits any non-commercial use, distribution, adaptation, and reproduction provided the original author and source are given appropriate credit. (C) The Author 2018

Received: 28 November 2018

Published Online: 21 December 2018

Citation: European Ophthalmic Review. 2018;12(2):83-4

Corresponding Author: Noel Alpins, 7 Chesterville

Road, Cheltenham VIC 3192, Australia.

E: alpins@newvisionclinics.com.au

Support: No funding was received in

the publication of this article.

\section{Ocular residual astigmatism}

The initial step of planning any refractive laser treatment that requires correction of astigmatism is to calculate the difference in magnitude and axes between the refractive cylinder at the corneal plane, and the corneal astigmatism - that is the vectorial difference. This has been defined as the ocular residual astigmatism (ORA) and is expressed in dioptres (D). ${ }^{.}$When calculating the ORA, the refractive cylinder needs to be converted to a positive magnitude at the corneal plane to allow accurate comparison to the corneal astigmatism. Several values of the ORA can be calculated for any one eye depending on which readings have been used for corneal astigmatism and which for refractive cylinder. Typically, the refractive cylinder and axis, as measured by the manifest refraction, is used for the refractive astigmatism parameter, although the cycloplegic, ocular wavefront or automated refraction can be used. The same applies for various measures of corneal astigmatism. Again, typically this is measured using a topographer, or more accurately with a tomographer, to include the posterior cornea, but one can also use automated keratometry from partial coherence interferometry devices, manual keratometry or corneal topographic astigmatism (CorT) which has been shown to be a more accurate measure of corneal astigmatism than simulated keratometry, manual keratometry and autokeratometry.,3 The combination of refractive and corneal parameters that result in the lowest magnitude of ORA preoperatively are typically the best matched and therefore the most accurate. The average ORA lies somewhere between $0.73 \mathrm{D}^{4}$ and 0.81 D. ${ }^{1}$ In more irregular corneas, such as keratoconus, the mean ORA has been calculated as $1.34 \mathrm{D},{ }^{5}$ somewhat higher than regular corneas. An ORA of $1.00 \mathrm{D}$ or greater is deemed to be significant and considered a red flag. The ORA will enable the surgeon to determine preoperatively whether all the astigmatism can be corrected or whether a residual amount will remain postoperatively on the cornea, potentially causing a suboptimal visual outcome. Subsequently, the patient can be advised on the expected outcome of refractive laser surgery. In cases where the treatment is predominantly astigmatic, for example plano/-2.00 x 75 on a near spherical cornea, and the ORA is high, then treatment by refractive parameters alone should be avoided and Vector Planning ${ }^{\circledR}$ (ASSORT Pty. Ltd., Cheltenham, Victoria, Australia) used as the preferred method as this will optimise the amount of astigmatism that can be treated and left remaining on the cornea.

\section{Corneal topographic astigmatism}

CorT is an accurate measure of corneal astigmatism based on all the measured data acquired from topography or tomography, and is calculated using the iASSORT ${ }^{\circledR}$ software (ASSORT Pty. Ltd., Cheltenham, Victoria, Australia; www.assort.com). CorT anterior is calculated using the front surface of the cornea only, based on topography, while CorT total incorporates the posterior cornea into the calculation for total corneal power using a tomographer for acquisition. ${ }^{2,3}$

\section{Vector Planning}

Using the example in Figure 1, the refractive cylinder (corneal plane) is $-1.59 \mathrm{DC} \times 15$ and the corneal astigmatism from CorT total is $2.80 \mathrm{D} @ 95$. The ORA is calculated as $1.41 \mathrm{D}$ Ax 174. Once the ORA has been determined, what can be done about it? Ideally, when the magnitude and axis 
Figure 1: Manifest refraction cylinder parameters versus corneal topographic astigmatism to calculate the ocular residual astigmatism

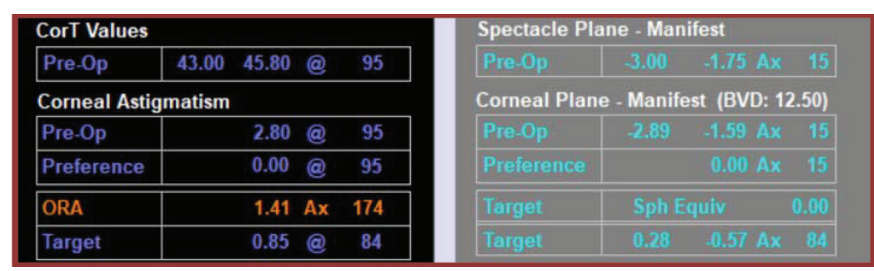

$B V D=$ back vertex distance; CorT $=$ corneal topographic astigmatism;

$O R A=$ ocular residual astigmatism; Pre-Op = pre-operation .

Figure 2: Treatments based on refractive cylinder parameters alone leave all the ocular residual astigmatism on the cornea postoperatively

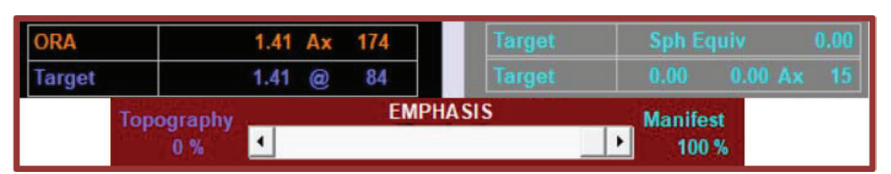

Ax = axis of cylindric lens; ORA = ocular residual astigmatism;

Sph equiv $=$ spherical equivalent.

of the corneal and refractive astigmatism are the same, which is rare, then the ORA is zero and treating by refractive or corneal parameters alone would be appropriate. However, in most cases where this is not the case, treating by refractive parameters alone compared to incorporating vector Planning will leave an excess amount of astigmatism on the cornea; that is, all the ORA (1.41 D) will remain on the cornea postoperatively (Figure 2). Conversely, treating by corneal astigmatism (topography-guided alone) will leave all the ORA in the refraction (Figure 3), often leaving the patient still requiring spectacle correction. The method of Vector Planning allows the surgeon to systematically treat the astigmatism anywhere on the 99 percentage points in between the two extremes of zero and $100 \%$, with the aim of minimising the corneal and refractive astigmatism postoperatively and leaving the corneal astigmatism in a favourable and acceptable orientation such as with-the-rule. Previous studies have found the 'sweet spot' to lie at $60 \%$ emphasis on the ORA by refraction and $40 \%$ according to corneal parameters, 5,6 however, this can be customised by the surgeon on a case-by-case basis, the preferred range lying between 50-65\% to refraction.

It is important to note that the target corneal astigmatism (0.85 D @ 84) is aligned $90^{\circ}$ away from the axis of the ORA (1.41 D Ax 174) to neutralise it, leaving the target refractive cylinder $(-0.57 \mathrm{DC}$ Ax 84$)$ at the same positive axis as the ORA (Figure 1) to neutralise it. The target refraction is set to a spherical equivalent of zero, unless targeting monovision, so that the corneal astigmatism is reduced compared to treating by refractive cylinder parameters alone without increasing the refractive cylinder in practice. So not only is the corneal astigmatism reduced but the refractive cylinder is also reduced or the same as when treating by $100 \%$ refractive parameters. This can be related to the benefit of having a better shaped cornea, with less coma and trefoil, and with fewer complaints of associated symptoms.

The method of Vector Planning also incorporates a safety mechanism whereby if the corneal astigmatism and the refractive cylinder are quite
Figure 3: Treatments based on corneal astigmatism parameters alone leave all the ocular residual astigmatism in the refraction postoperatively

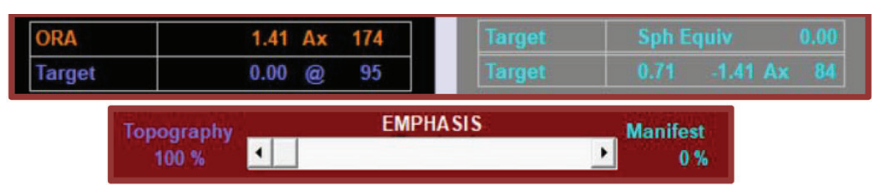

ORA = ocular residual astigmatism; Sph equiv = spherical equivalent .

Figure 4: Refractive and corneal astigmatism that are disparate in orientation lead to a high ocular residual astigmatism

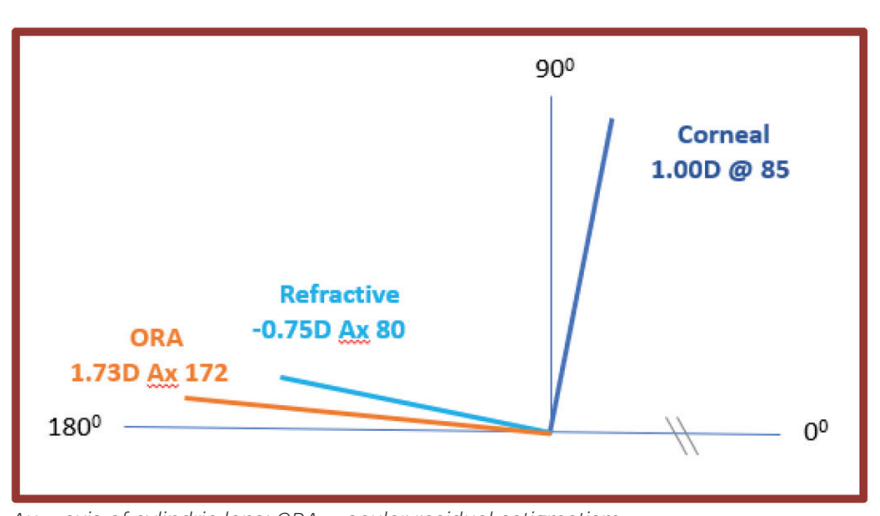

Ax = axis of cylindric lens; ORA = ocular residual astigmatism.

disparate (for example, the corneal astigmatism is $1.00 \mathrm{D} @ 85$ and the refractive cylinder is $-0.75 \mathrm{D}$ Ax 80; Figure 4), then treating a minimum amount of astigmatism or not treating any astigmatism at all will be planned, as treating either refractive or corneal astigmatism in preference will cause an excess of the other with a potential adverse effect. This is to avoid increasing the astigmatism magnitude postoperatively compared to the preoperative magnitude. There have been several studies using Vector Planning in refractive laser surgery, 5,6 with the most recent one from Oman being the most significant in terms of a large cohort.? The study by Dr Maria Arbelaez et al. used the Schwind Amaris excimer laser to treat 80 eyes using manifest refraction alone and 80 eyes with Vector Planning. Postoperatively the Vector Planning group had better visual results and less corneal astigmatism with similar refractive cylinder outcomes compared to the manifest refraction group?

It is essential that, as corneal surgeons, we plan refractive laser procedures with the corneal as well as the refractive parameters in the treatment plan. Better measures of corneal astigmatism are now available to make its inclusion safe. After all, when planning toric intraocular lens procedures, it is the corneal astigmatism that is paramount in the selection of the most accurate implant to optimise astigmatic outcomes. Excimer laser refractive surgery similarly has a high priority for favourable corneal outcomes with a plano refractive result.

The vector Planning calculator is freely available for trial on the ASSORT ${ }^{\circledast}$ website (www.assort.com).
Alpins NA. New method of targeting vectors to treat astigmatism. J Cataract Refract Surg. 1997:23:65-75.

Alpins NA, Ong JK, Stamatelatos G. New method of quantifying corneal topographic astigmatism that corresponds with manifest refractive cylinder. J Cataract Refract Surg. 2012;38:1978-88

Alpins NA, Stamatelatos G, Ong J. Corneal topographic

astigmatism (CorT) to quantify total corneal astigmatism. Journal Refract Surg. 2015;31:182-6.
4. Alpins NA. Astigmatism analysis by the Alpins method. J Cataract Refract Surg. 2001;27:31-49.

Alpins NA, Stamatelatos G. Customized photoastigmatic refractive keratectomy using combined topographic and refractive data for myopia and astigmatism in refractive data for myopia and astigmatism in eyes with 2007:33:591-602.

6. Alpins NA, Stamatelatos G. Clinical Outcomes for laser in situ keratomileusis using combined topography and refractive wavefront treatments for myopic astigmatism. J Cataract Refract Surg. 2008:34:1250-9.

Arbelaez MC, Alpins N, Verma S, et al. Clinical outcomes of laser in situ keratomileusis with an aberration-neutral profile laser in situ keratomileusis wis an aberation neutral profile centered on the corneal vertex comparing vector planning with manifest refraction planng for the treatment of my 